Pure and Applied Mathematics Quarterly

Volume 5, Number 1

(Special Issue: In honor of

Jean-Pierre Serre, Part 2 of 2)

$349-374,2009$

\title{
Discriminant of Symplectic Involutions
}

\author{
Skip Garibaldi, R. Parimala and Jean-Pierre Tignol \\ à Jean-Pierre Serre, pour son $80^{e}$ anniversaire
}

\begin{abstract}
We define an invariant of torsors under adjoint linear algebraic groups of type $C_{n}$ - equivalently, central simple algebras of degree $2 n$ with symplectic involution - for $n$ divisible by 4 that takes values in $H^{3}\left(F, \mu_{2}\right)$. The invariant is distinct from the few known examples of cohomological invariants of torsors under adjoint groups. We also prove that the invariant detects whether a central simple algebra of degree 8 with symplectic involution can be decomposed as a tensor product of quaternion algebras with involution.
\end{abstract}

Keywords: cohomological invariants, symplectic groups, central simple algebras with involution.

\section{INTRODUCTION}

While the Rost invariant is a degree 3 invariant defined for torsors under simply connected simple algebraic groups, there are very few degree 3 invariants known for adjoint groups. In this paper, we define such an invariant for torsors under adjoint algebraic groups of symplectic type and show that this invariant gives a

Received July 6, 2007.

2000 Mathematics Subject Classification. 11E72 (16W10).

The authors were partially supported by NSF grant DMS-0654502, NSF grant DMS-0653382, and the Fund for Scientific Research - FNRS (Belgium), respectively. The third author would like to thank his co-authors and Emory University for their hospitality while the work for this paper was carried out. 
necessary and sufficient condition for decomposability of symplectic involutions on degree 8 algebras. This decomposability criterion is analogous to the criteria given for degree 4 algebras with orthogonal involutions in [KPS] and for unitary involutions from $[\mathrm{KQ}]$.

In the paper $[\mathrm{BMT}]$, the authors defined a "relative" invariant of symplectic involutions: for $\sigma, \tau$ symplectic involutions on the same central simple algebra $A$ (defined over a field $F$ of characteristic $\neq 2$ and of degree divisible by 4 ), they defined

$$
\Delta_{\tau}(\sigma)=(\operatorname{Nrp}(s)) \cdot[A] \quad \in H^{3}\left(F, \mu_{2}\right)
$$

where $s$ is a $\tau$-symmetric element of $A^{\times}$such that $\operatorname{Int}(s) \tau=\sigma$. (Alternatively, $\Delta_{\tau}$ can be defined by pushing the Rost invariant of the simply connected group $\operatorname{Sp}(A, \tau)$ forward along the natural projection to the adjoint group.) If the index of $A$ divides half the degree, then one gets an "absolute" invariant by taking $\tau$ to be hyperbolic.

Our first result shows that the relative invariant defined in [BMT] leads to an absolute invariant of all symplectic involutions on algebras of a fixed degree, i.e., to a cohomological invariant of the split adjoint group $\mathrm{PGSp}_{2 n}$ of type $C_{n}$ for $n$ divisible by 4 . Recall that the set $H^{1}\left(K, \mathrm{PGSp}_{2 n}\right)$ classifies central simple $K$-algebras of degree $2 n$ endowed with a symplectic involution [KMRT, 29.22] for each extension $K / F$, and this defines a functor Fields $/ F \rightarrow$ Sets. The map $K \mapsto H^{3}\left(K, \mu_{2}\right)$ also defines a functor Fields $/ F \rightarrow$ Sets, and an invariant $H^{1}\left(*, \mathrm{PGSp}_{2 n}\right) \rightarrow H^{3}\left(*, \mu_{2}\right)$ (in the sense of [GMS]) is a morphism of functors, i.e., a map $a_{K}: H^{1}\left(K, \mathrm{PGSp}_{2 n}\right) \rightarrow H^{3}\left(K, \mu_{2}\right)$ for every extension $K / F$ together with a compatibility condition.

Theorem A. For every $n$ divisible by 4 , there is a unique invariant

$$
\Delta: H^{1}\left(*, \mathrm{PGSp}_{2 n}\right) \rightarrow H^{3}\left(*, \mu_{2}\right)
$$

such that for every extension $K / F$, we have:

(1) If $(A, \sigma)$ is a central simple $K$-algebra with hyperbolic symplectic involution, then $\Delta(A, \sigma)=0$.

(2) If $\sigma, \tau$ are symplectic involutions on the same $K$-algebra $A$, then

$$
\Delta_{\tau}(\sigma)=\Delta(A, \sigma)-\Delta(A, \tau) .
$$


Property (1) includes the statement that $\Delta_{K}$ sends zero to zero for each extension $K / F$, i.e., $\Delta$ is normalized in the sense of [GMS]. Property (2) can be replaced by " $\Delta$ is nonzero", see 4.5 below.

No such invariant $\Delta$ exists in case $n$ is not divisible by 4 , see Prop. 4.1 for a precise statement.

We say that $(A, \sigma)$ is completely decomposable if there are quaternion algebras $Q_{i}$ endowed with involutions $\sigma_{i}$ of the first kind such that $(A, \sigma)$ is isomorphic to the tensor product $\otimes\left(Q_{i}, \sigma_{i}\right)$. For $\sigma_{1}, \sigma_{2}$ orthogonal, the tensor product $\left(Q_{1}, \sigma_{1}\right) \otimes$ $\left(Q_{2}, \sigma_{2}\right)$ is isomorphic to a tensor product $\left(Q_{1}^{\prime}, \gamma_{1}\right) \otimes\left(Q_{2}^{\prime}, \gamma_{2}\right)$ where $Q_{1}^{\prime}, Q_{2}^{\prime}$ are quaternion algebras and $\gamma_{1}, \gamma_{2}$ are the canonical symplectic involutions [KPS, 5.2]. Thus every completely decomposable $(A, \sigma)$ can be written as a tensor product of quaternion algebras with symplectic involutions and at most one quaternion algebra with orthogonal involution.

It follows from results in the literature that $\Delta$ vanishes on completely decomposable involutions, see Example 3.2. Our second theorem shows that the converse holds for algebras of degree 8 :

Theorem B. Let $A$ be a central simple algebra of degree 8 with symplectic involution $\sigma$. The element $\Delta(A, \sigma)$ is zero if and only if $(A, \sigma)$ is completely decomposable.

Finally, we address the Pfister Factor Conjecture from [Sh], which asserts: Let $\left(Q_{i}, \sigma_{i}\right)$ be quaternion $F$-algebras endowed with orthogonal involutions for $1 \leq i \leq$ $n$. Over every extension $K / F$ such that the tensor product $Q_{1} \otimes Q_{2} \otimes \cdots \otimes Q_{n}$ is split, the involution $\sigma_{1} \otimes \sigma_{2} \otimes \cdots \otimes \sigma_{n}$ is adjoint to a Pfister form. This conjecture is now known in general by [Be]. We use our invariant to give an alternate proof for $n=6$.

Notation and conventions. We assume throughout the paper that the characteristic of $F$ is different from 2 .

We sometimes write ${ }^{-}$for the standard (symplectic) involution on a quaternion algebra. For $g$ an element of a group and $h$ an endomorphism of the group, we write $\operatorname{Int}(g) h$ for the map $x \mapsto g h(x) g^{-1}$.

For an algebraic group $G$ over $F$, we write $H^{d}(F, G)$ for the Galois cohomology set $H^{d}\left(\operatorname{Gal}\left(F_{\text {sep }} / F\right), G\left(F_{\text {sep }}\right)\right)$ where $F_{\text {sep }}$ denotes a separable closure of $F$. The 
group $H^{1}\left(F, \mu_{2}\right)$ is identified with $F^{\times} / F^{\times 2}$ by Kummer theory, and we write $(x)$ for the element of $H^{1}\left(F, \mu_{2}\right)$ corresponding to $x F^{\times 2}$. The group $H^{2}\left(F, \mu_{2}\right)$ is identified with the 2-torsion in the Brauer group of $F$, and we write $[A]$ for the element of $H^{2}\left(F, \mu_{2}\right)$ corresponding to a central simple algebra $A$ of exponent 2 .

General background on these topics and on algebras with involution can be found in $[\mathrm{KMRT}]$. For the Rost invariant, see [GMS].

\section{Proof of Theorem A}

This section consists of a proof of Theorem A.

(i): We first prove uniqueness. Let $\Delta, \Delta^{\prime}$ be invariants as in the theorem, and consider $(A, \sigma) \in H^{1}\left(K, \mathrm{PGSp}_{2 n}\right)$. In the Brauer group, the class of $A$ can be written as a sum $\sum_{i=1}^{\ell}\left[Q_{i}\right]$ of quaternion algebras, and the symbol length of $A$ is defined to be the minimum such $\ell$. We prove that $\Delta$ equals $\Delta^{\prime}$ by induction on $\ell$.

If $\ell$ is 0 or 1 , i.e., if $A$ has index 1 or 2 , then applying (1) and (2) to compare $\sigma$ with the hyperbolic involution $\tau$ on $A$ shows that

$$
\Delta(A, \sigma)=\Delta_{\tau}(\sigma)+0=\Delta^{\prime}(A, \sigma) .
$$

If $\ell$ is at least 2, then we let $E$ be the function field of the Albert quadric for the product $Q_{1} \otimes Q_{2}$. Recall from, say, [KMRT, 16.4, 16.5] that if $Q_{i}=\left(a_{i}, b_{i}\right)$ for $a_{i}, b_{i} \in F^{\times}$, the Albert quadric of $Q_{1} \otimes Q_{2}$ is the variety defined by the quadratic polynomial

$$
a_{1} X_{1}^{2}+b_{1} X_{2}^{2}-a_{1} b_{1} X_{3}^{2}-a_{2} X_{4}^{2}-b_{2} X_{5}^{2}+a_{2} b_{2} X_{6}^{2}
$$

and that $Q_{1} \otimes Q_{2} \otimes E$ has index 2. Then $A \otimes E$ has symbol length strictly less than $\ell$, hence $\Delta$ and $\Delta^{\prime}$ agree on $\operatorname{res}_{E / K}(A, \sigma)$. However, the restriction map $H^{3}\left(K, \mu_{2}\right) \rightarrow H^{3}\left(E, \mu_{2}\right)$ is injective [A, 5.6], so $\Delta$ and $\Delta^{\prime}$ agree on $(A, \sigma)$.

(ii): We prove the existence of $\Delta$ in the same manner as uniqueness, i.e., by induction on the symbol length $\ell$, supposing at each stage that $\Delta$ is defined for every algebra of symbol length $<\ell$ over every extension of $F$ and satisfies (1) and (2) and is functorial.

For $\ell \leq 2$, the definition is clear: A central simple algebra $A$ of degree $2 n$ that can be written as a sum of at most 2 quaternion algebras has index 1, 2, 
or 4 . As $n$ is divisible by 4 , there is a hyperbolic involution $\tau$ on $A$ and we put $\Delta(A, \sigma):=\Delta_{\tau}(\sigma)$. This defines an invariant satisfying properties (1) and (2) by the results of $[\mathrm{BMT}]$.

(iii): Suppose that $\ell$ is at least 3. Let $X$ be the Albert quadric for the biquaternion algebra $Q_{1} \otimes Q_{2}$. We put:

$$
\delta:=\Delta((A, \sigma) \otimes F(X)) \quad \in H^{3}\left(F(X), \mu_{2}\right)
$$

where the right side is defined because $A \otimes F(X)$ has symbol length strictly less than $\ell$ in the Brauer group of $F(X)$. We claim that $\delta$ belongs to the image of $H^{3}\left(F, \mu_{2}\right) \rightarrow H^{3}\left(F(X), \mu_{2}\right)$.

We first verify that $\delta$ is unramified. Let $x$ be a codimension 1 point of $X$, write $F(x)$ for its residue field, and write $K$ for the completion of $F(X)$ at $x$. By Cohen's Structure Theorem, there is a finite purely inseparable extension $K_{1}$ of $K$ with residue field $F_{1}$ such that there is an $F$-embedding $F_{1} \hookrightarrow K_{1}$ splitting the residue map, see e.g. [GMS, p. 30]. We obtain a commutative diagram [GMS, p. 19]:

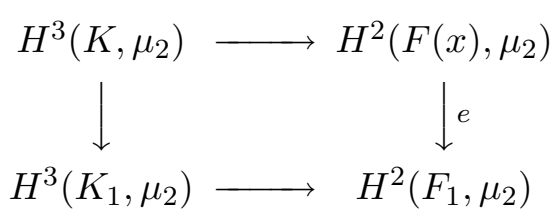

where $e$ denotes the ramification index of $K_{1} / K$. The element $\Delta((A, \sigma) \otimes F(x))$ of $H^{3}\left(F(x), \mu_{2}\right)$ is well defined (because the symbol length of $A$ is lower over $F(x)$ ) and has the same image as $\delta$ in $H^{3}\left(K_{1}, \mu_{2}\right)$ (via the embedding $F(x) \hookrightarrow K_{1}$ ). Hence $\delta$ is unramified over $K_{1}$, i.e., $\delta$ in the upper left corner maps to zero in the lower right. As $K_{1} / K$ is a purely inseparable extension, it has odd degree, so the vertical arrows are injections and the residue of $\delta$ in $H^{2}\left(F(x), \mu_{2}\right)$ is zero. We have shown that $\delta$ belongs to the subgroup $H_{\mathrm{nr}}^{3}\left(F(X), \mu_{2}\right)$ of $H^{3}\left(F(X), \mu_{2}\right)$ consisting of unramified classes.

Let now $L$ be a generic splitting field for $Q_{\ell}$. We claim that $X$ remains anisotropic over $L$ (because $\ell \neq 1,2$ ). Otherwise, by [Lam, XIII.2.13], $Q_{1} \otimes Q_{2}$ is isomorphic to $H \otimes Q_{\ell}$ for some quaternion $F$-algebra $H$. But in that case the Brauer class of $A$ equals $[H]+\sum_{i=3}^{\ell-1}\left[Q_{i}\right]$, contradicting the definition of $\ell$. 
By [Kahn], we have a diagram with exact rows:

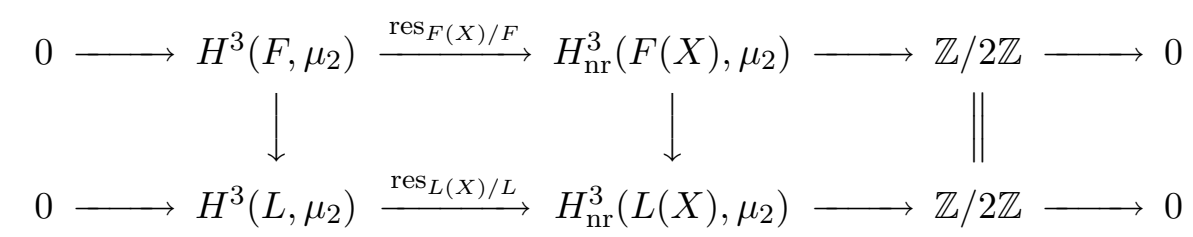

Since $X$ is anisotropic over $F$ and $L$, the diagram commutes by the explicit description of an element of $H_{\mathrm{nr}}^{3}\left(F(X), \mu_{2}\right)$ mapping to the nonzero element in $\mathbb{Z} / 2 \mathbb{Z}$ from [Kahn, p. 249]. The symbol lengths of $A$ over $F(X)$ and over $L$ are at most $\ell-1$, so we have:

$$
\Delta\left(\left[(A, \sigma) \otimes_{F} F(X)\right] \otimes_{F(X)} L(X)\right)=\Delta\left(\left[(A, \sigma) \otimes_{F} L\right] \otimes_{L} L(X)\right),
$$

that is,

$$
\operatorname{res}_{L(X) / F(X)}(\delta)=\Delta((A, \sigma) \otimes L(X))=\operatorname{res}_{L(X) / L} \Delta((A, \sigma) \otimes L) .
$$

A diagram chase shows that $\delta$ is in the image of the restriction map $H^{3}\left(F, \mu_{2}\right) \rightarrow$ $H_{\mathrm{nr}}^{3}\left(F(X), \mu_{2}\right)$. We define $\Delta(A, \sigma) \in H^{3}\left(F, \mu_{2}\right)$ to be the unique element that maps to $\delta$.

(iv): Using the method of (i), one can verify properties (1) and (2) and functoriality for $\Delta$ relative to algebras of symbol length at most $\ell$ by induction. This completes the proof of existence of $\Delta$, hence of Theorem A.

Remark 2.2. In part (iii) of the proof above, the condition that $\ell \geq 3$ is necessary to prove that $\delta$ lies in the image of $H^{3}\left(F, \mu_{2}\right)$. By way of contrast, consider the case where $(A, \sigma)$ is a central simple $F$-algebra of degree 4 with symplectic involution; this case is not covered by Theorem $\mathrm{A}$. The algebra $A$ is a tensor product of quaternion algebras $Q_{1} \otimes Q_{2}$. Over the function field $F(X)$ of the corresponding Albert quadric, the algebra is isomorphic to 2-by-2 matrices over a quaternion algebra $H$ and $\sigma$ is adjoint to a hermitian form $\langle 1,-c\rangle$ with respect to the canonical involution on $H$. By [BMT, Example 3], the relative invariant with respect to the hyperbolic form $\tau$ on $M_{2}(H)$ is

$$
\Delta_{\tau}(\sigma)=(c) \cdot[H] \quad \in H^{3}\left(F(X), \mu_{2}\right) .
$$

This is an unramified class that is not in the image of $H^{3}\left(F, \mu_{2}\right)$ [Kahn, p. 249], i.e., it represents the nonzero element in the cokernel $\mathbb{Z} / 2 \mathbb{Z}$ from (2.1). 


\section{ExAmples}

Example 3.1. Let $B$ be a central simple algebra of degree divisible by 4 , endowed with an orthogonal involution $\rho$, and let $Q$ be a quaternion algebra. We have:

$$
\Delta\left[\left(Q,^{-}\right) \otimes(B, \rho)\right]=[Q] \cdot(\operatorname{disc} \rho),
$$

cf. [ST]. This can be proved by induction on the symbol length $\ell$ of $B$. If $\ell$ is 0 or 1 , then $B$ is not division and the lemma is [BMT, Example 3]. The case of larger $\ell$ is reduced to the case $\ell=1$ by the Albert quadric technique as in the proof of Theorem A.

Example 3.2. Suppose that $(A, \sigma)$ is completely decomposable as defined in the introduction, i.e., isomorphic to $\otimes_{i=1}^{n}\left(Q_{i}, \sigma_{i}\right)$, and $n$ is at least 3. By [KPS, 5.2] we can write $(A, \sigma)$ as in Example 3.1 with $(B, \rho)$ completely decomposable. Then $\operatorname{disc} \rho=1$ by [KMRT, 7.3], which gives: If $(A, \sigma)$ is completely decomposable and the degree of $A$ is at least 8 , then $\Delta(A, \sigma)$ is zero.

Example 3.3. Let $B$ be a central simple algebra of degree divisible by 4 endowed with a symplectic involution $\sigma$, and let $Q$ be a quaternion algebra. For every orthogonal involution $\rho$ on $Q$,

$$
\Delta[(Q, \rho) \otimes(B, \sigma)]=0 .
$$

To prove this, we use induction on the symbol length of $B$ and the Albert quadric technique to reduce to the case where $B$ is split or has index 2 . When $B$ is split, $\sigma$ and $\rho \otimes \sigma$ are hyperbolic and the formula is clear. If $B$ is Brauer-equivalent to a quaternion algebra $H$, then Proposition 3.4 of [BST] yields a decomposition

$$
(B, \sigma) \simeq\left(H,^{-}\right) \otimes\left(M_{m}(F), \tau\right)
$$

for some orthogonal involution $\tau$. By Example 3.1 we have

$$
\Delta[(Q, \rho) \otimes(B, \sigma)]=[H] \cdot(\operatorname{disc} \rho \otimes \tau)=0,
$$

as desired.

Proposition 3.4. If $(A, \sigma)=\left(B, \tau_{1}\right) \boxplus\left(B, \tau_{2}\right)$ for some central simple algebra $B$ of degree divisible by 4 and with symplectic involutions $\tau_{1}, \tau_{2}$, then $\Delta(A, \sigma)=$ $\Delta_{\tau_{1}}\left(\tau_{2}\right)$. 
Proof. Let $\hat{\tau}_{1}=t \otimes \tau_{1}$ on $M_{2}(B)=M_{2}(F) \otimes B$. The hypothesis means that we may identify

$$
A=M_{2}(B), \quad \sigma=\operatorname{Int}\left(\begin{array}{ll}
1 & 0 \\
0 & v
\end{array}\right) \hat{\tau}_{1}
$$

for some $v \in B^{\times}$such that $\tau_{2}=\operatorname{Int}(v) \tau_{1}$. Then

$$
\Delta(A, \sigma)-\Delta\left(A, \hat{\tau}_{1}\right)=\Delta_{\sigma}\left(\hat{\tau}_{1}\right)=\left(\operatorname{Nrp}_{\sigma}\left(\begin{array}{ll}
1 & 0 \\
0 & v
\end{array}\right)\right) \cdot[A]=\left(\operatorname{Nrp}_{\tau_{1}}(v)\right) \cdot[B]=\Delta_{\tau_{1}}\left(\tau_{2}\right) .
$$

On the other hand, $\left(A, \hat{\tau}_{1}\right)$ can be decomposed as $\left(M_{2}(F), t\right) \otimes\left(B, \tau_{1}\right)$, so $\Delta\left(A, \hat{\tau}_{1}\right)$ is zero by Example 3.3 .

\section{Degree 3 invariants of PGSp$_{2 n}$}

Consider the following invariants that map $H^{1}\left(*, \mathrm{PGSp}_{2 n}\right) \rightarrow H^{d}\left(*, \mu_{2}\right)$ for various $d$ :

(1) The constant invariant $\underline{1}$ that sends every $(A, \sigma) \in H^{1}\left(K, \mathrm{PGSp}_{2 n}\right)$ to the 1 in the ring $H^{\bullet}\left(K, \mu_{2}\right)$.

(2) The invariant $\delta: H^{1}\left(*, \mathrm{PGSp}_{2 n}\right) \rightarrow H^{2}\left(*, \mu_{2}\right)$ that is given by the connecting homomorphism arising from the surjection $\pi: \mathrm{Sp}_{2 n} \rightarrow \mathrm{PGSp}_{2 n}$, where $\mathrm{Sp}_{2 n}$ denotes the simply connected cover of PGSp ${ }_{2 n}$. The map $\delta_{K}$ sends $(A, \sigma) \in H^{1}\left(K, \mathrm{PGSp}_{2 n}\right)$ to the class $[A]$ in $H^{2}\left(K, \mu_{2}\right)$.

(3) The invariant $\Delta$ defined in Th. A; it is defined when $n$ is divisible by 4 .

The collection of invariants $H^{1}\left(*, \mathrm{PGSp}_{2 n}\right) \rightarrow H^{\bullet}\left(*, \mu_{2}\right)$ is a module over the ring $H^{\bullet}\left(F, \mu_{2}\right)$, and the invariants $\underline{1}, \delta, \Delta$ are linearly independent over that ring. We prove the following spanning result:

Proposition 4.1. Suppose that $n$ is even. For $d$ at most 3, every invariant $H^{1}\left(*, \mathrm{PGSp}_{2 n}\right) \rightarrow H^{d}\left(*, \mu_{2}\right)$ is in the span of $\underline{1}, \delta, \Delta$ if $n$ is divisible by 4 , and in the span of $\underline{1}, \delta$ otherwise.

Proof. (i): For $d=1,2$, the proposition is contained in [KMRT, 31.15, 31.20], so we consider the case $d=3$. Let $a$ be an invariant $H^{1}\left(*, \mathrm{PGSp}_{2 n}\right) \rightarrow H^{3}\left(*, \mu_{2}\right)$. Consider the functor $H^{1}\left(*, \mathrm{PGL}_{2}\right) \rightarrow H^{1}\left(*, \mathrm{PGSp}_{2 n}\right)$ that sends a quaternion algebra $Q$ to the algebra $M_{n}(Q)$ endowed with a hyperbolic involution (which exists because $n$ is even); composing this with $a$, we obtain an invariant $H^{1}\left(*, \mathrm{PGL}_{2}\right) \rightarrow$ $H^{3}\left(*, \mu_{2}\right)$ that is necessarily of the form

$$
Q \mapsto \lambda_{3}+\lambda_{1} \cdot[Q]
$$


for uniquely determined $\lambda_{3} \in H^{3}\left(F, \mu_{2}\right)$ and $\lambda_{1} \in H^{1}\left(F, \mu_{2}\right)$ by [GMS, p. 43].

Let $(A, \sigma)$ denote a versal $\mathrm{PGSp}_{2 n}$-torsor defined over a field $K$. Write $\psi_{E}$ for the composition

$$
H^{1}(E, \operatorname{Sp}(A, \sigma)) \stackrel{\pi}{\longrightarrow} H^{1}(E, \operatorname{PGSp}(A, \sigma)) \underset{\text { twist }}{\sim} H^{1}\left(E, \operatorname{PGSp}_{2 n}\right) \stackrel{a_{E}}{\longrightarrow} H^{3}\left(E, \mu_{2}\right)
$$

for extensions $E$ of $K$; this defines an invariant $\psi$ of torsors under $\operatorname{Sp}(A, \sigma)$. By [GMS, p. 127] this invariant is of the form

$$
\eta \mapsto \lambda_{0} r_{E}(\eta)+a_{E}(A \otimes E, \sigma)
$$

for fixed $\lambda_{0}$ equal to 0 or 1 , where $r$ denotes the Rost invariant $H^{1}(*, \operatorname{Sp}(A, \sigma)) \rightarrow$ $H^{3}\left(*, \mu_{2}\right)$. Recall from [KMRT, 29.24] that the set $H^{1}(E, \operatorname{Sp}(A, \sigma))$ is identified with a quotient of the set of $\sigma$-symmetric elements $s \in(A \otimes E)^{\times}$. For such an $s$, we have by [KMRT, 31.46]:

$$
\psi_{E}(s)=\lambda_{0} \cdot(\operatorname{Nrp}(s)) \cdot[A]+a_{E}(A \otimes E, \sigma),
$$

which is $a_{E}(A \otimes E, \operatorname{Int}(s) \tau)$ by the definition of $\psi$. Whence, for every extension $E / K$ and every symplectic involution $\tau$ on $A \otimes E$, we have

$$
a_{E}(A \otimes E, \sigma)-a_{E}(A \otimes E, \tau)=\lambda_{0} \cdot \Delta_{\tau}(\sigma) .
$$

(ii): Suppose first that $n$ is divisible by 4 . We claim that $a$ is the invariant $a^{\prime}:=\lambda_{3}+\lambda_{1} \cdot \delta+\lambda_{0} \cdot \Delta$. By [GMS, p. 31], it suffices to check that $a$ and $a^{\prime}$ take the same value on the versal torsor $(A, \sigma) \in H^{1}\left(K, \mathrm{PGSp}_{2 n}\right)$. Arguing as in the proof of Th. A above, we may find an extension $E / K$ such that $A \otimes E$ has index 2 and the restriction $H^{3}\left(K, \mu_{2}\right) \rightarrow H^{3}\left(E, \mu_{2}\right)$ is injective, so it suffices to prove that $a$ and $a^{\prime}$ agree on $\operatorname{res}_{E / K}(A, \sigma) \in H^{1}\left(E, \mathrm{PGSp}_{2 n}\right)$. As $A \otimes E$ has index 2, it supports a hyperbolic involution $\tau$. By construction (and property (1) of $\Delta$ ), the invariants $a$ and $a^{\prime}$ both take the value $\lambda_{3}+\lambda_{1} \cdot[A]$ on $(A \otimes E, \tau)$. On the other hand, by property (2) of $\Delta$ we have:

$$
a_{E}^{\prime}(A \otimes E, \sigma)-a_{E}^{\prime}(A \otimes E, \tau)=\lambda_{0} \cdot \Delta_{\tau}(\sigma),
$$

which equals $a_{E}(A \otimes E, \sigma)-a_{E}(A \otimes E, \tau)$ by (4.3). Combining the two preceding sentences, we conclude that $a$ and $a^{\prime}$ agree on $(A \otimes E, \sigma)$, hence are the same invariant. 
(iii): Suppose now that $n=2 m$ for some $m$ odd. Replacing $a$ with $a-\left(\lambda_{3}+\right.$ $\lambda_{1} \cdot \delta$ ), we may assume that $a$ vanishes on hyperbolic involutions. (Note that an algebra of degree $4 m$ with hyperbolic involution necessarily has index 1 or 2.) We want to show that $a$ is the zero invariant.

We claim that the element $\lambda_{0}$ defined in (i) is 0. The algebra $A$ is Brauerequivalent to a biquaternion division $K$-algebra $B$. Fix a symplectic involution $\gamma$ on $B$ and define an involution $\theta$ on $A$ via

$$
(A, \theta) \cong\left(M_{m}(K), t\right) \otimes(B, \gamma) .
$$

Let $X$ be the Albert quadric for $B$, and fix a hyperbolic symplectic involution $\tau$ on $A \otimes K(X)$. Because $a$ vanishes on hyperbolic involutions, we have:

$$
\begin{aligned}
\operatorname{res}_{K(X) / K} a(A, \theta)= & a(A \otimes K(X), \theta) \\
= & a(A \otimes K(X), \theta)-a(A \otimes K(X), \sigma) \\
& +a(A \otimes K(X), \sigma)-a(A \otimes K(X), \tau) .
\end{aligned}
$$

Applying (4.3) twice, we find:

$$
\operatorname{res}_{K(X) / K}(A, \theta)=\lambda_{0} \cdot\left(\Delta_{\sigma}(\theta)+\Delta_{\tau}(\sigma)\right)=\lambda_{0} \cdot \Delta_{\tau}(\theta),
$$

where the second equality is by [BMT, Prop. 1b].

The algebra $B \otimes K(X)$ is Brauer-equivalent to a quaternion division algebra $H$ over $K(X)$ and $\theta$ is adjoint to a hermitian form

$$
\langle 1,1, \ldots, 1\rangle \otimes\langle 1,-c\rangle
$$

(with respect to the canonical involution on $H$ ) for some $c \in K(X)^{\times}$. As in Remark 2.2, $\Delta_{\tau}(\theta)=(c) \cdot[H]$, which is an element of $H^{3}\left(K(X), \mu_{2}\right)$ that does not come from $H^{3}\left(K, \mu_{2}\right)$. As $a(A, \theta)$ is an element of $H^{3}\left(K, \mu_{2}\right)$, we conclude via (4.4) that $\lambda_{0}$ is zero.

By (4.3), we have:

$$
a(A \otimes K(X), \sigma)=a(A \otimes K(X), \tau)=0 .
$$

Hence $a(A, \sigma)$ is zero. As $(A, \sigma)$ is a versal $\mathrm{PGSp}_{2 n}$-torsor, this proves the proposition.

4.5. Suppose $n$ is divisible by 4 and $a$ is a nonzero invariant $H^{1}\left(*, \mathrm{PGSp}_{2 n}\right) \rightarrow$ $H^{3}\left(*, \mu_{2}\right)$ that satisfies condition (1) of Th. A, i.e., such that $a(A, \sigma)$ is zero if $\sigma$ 
is hyperbolic. It follows easily from Prop. 4.1 that $a$ equals $\Delta$. Therefore, in the statement of Th. A, condition (2) can be replaced with: $\Delta$ is nonzero.

Remark 4.6. In the case $n=2$, the exceptional isomorphism $\mathrm{C}_{2}=\mathrm{B}_{2}$ shows that $\mathrm{PGSp}_{2 n}$ is the split special orthogonal group on a 5 -dimensional space. The invariants $H^{1}\left(*, \mathrm{PGSp}_{4}\right) \rightarrow H^{\bullet}\left(*, \mu_{2}\right)$ were determined in [GMS, p. 44]; they make up a free $H^{\bullet}\left(F, \mu_{2}\right)$-module of rank 3 .

The invariants of $\mathrm{PGSp}_{2 n}$-torsors when $n$ is odd are treated by Mark MacDonald in $[\mathrm{Mac}]$.

\section{Alternative Definition of $\Delta$ For algebras of Degree 8}

In the case $n=4-$ corresponding to algebras of degree 8 - the invariant $\Delta$ given by Theorem A can be seen as a special case of the Rost invariant. Most of what is written here is not used in the rest of this paper, so we omit many details.

5.1. The inclusion $\mathrm{PGSp}_{8} \subset E_{6}$. We view the split adjoint group $\mathrm{PGSp}_{8}$ as a subgroup of the split simply connected group $E_{6}$ of that type. (This inclusion is well known, see e.g. [D, p. 193].) We view $E_{6}$ as a Chevalley group given in terms of generators and relations as in [St lect]. Let $\alpha_{1}, \alpha_{2}, \ldots, \alpha_{6}$ be simple roots of $E_{6}$ numbered as in the diagram

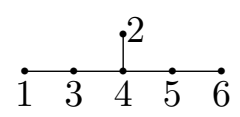

Write $h_{\alpha_{i}}: \mathbb{G}_{m} \rightarrow E_{6}$ for the homomorphism corresponding to $\alpha_{i}$ such that the images of the $h_{\alpha_{i}}$ generate a maximal torus in $E_{6}$. Write $\phi$ for the outer automorphism of $E_{6}$ that permutes the generators in a manner corresponding to the non-identity automorphism of the Dynkin diagram. The subgroup $H$ of $E_{6}$ consisting of elements fixed by the automorphism

$$
\operatorname{Int}\left(h_{\alpha_{1}}(-1) h_{\alpha_{4}}(-1) h_{\alpha_{6}}(-1)\right) \cdot \phi
$$

is connected and reductive [Stend, 8.1]. The root system of $H$ is explicitly described by the recipe from p. 275 of [St coll]; it is of type $C_{4}$.

Let $\mathrm{Sp}_{8}$ be the simply connected cover of $H$. Since it is simply connected, the cocharacter group of a maximal torus is identified with the coroot lattice. 
The same holds for $E_{6}$, and we can describe the arrow $\mathrm{Sp}_{8} \rightarrow E_{6}$ on the level of maximal tori by computing the corresponding map on coroot lattices. Number the simple roots of $C_{4}$ as $\gamma_{1}, \gamma_{2}, \gamma_{3}, \gamma_{4}$ as in the diagram

$$
\stackrel{i}{2} \quad \stackrel{3}{3} \dot{4}
$$

It follows from the explicit description of the root system of $H$ that the map of a maximal torus in $\mathrm{Sp}_{8}$ to one in $E_{6}$ is given by

$$
\begin{array}{ll}
\check{\gamma}_{1} \mapsto 2 \check{\alpha}_{2}+\check{\alpha}_{3}+2 \check{\alpha}_{4}+\check{\alpha}_{5} & \check{\gamma}_{2} \mapsto \check{\alpha}_{1}+\check{\alpha}_{6} \\
\check{\gamma}_{3} \mapsto \check{\alpha}_{3}+\check{\alpha}_{5} & \check{\gamma}_{4} \mapsto \check{\alpha}_{4}
\end{array}
$$

Notice that the short coroot $\check{\gamma}_{4}$ maps to the short coroot $\check{\alpha}_{4}$. By [GMS, p. 122], this implies:

The composition of $\mathrm{Sp}_{8} \rightarrow E_{6}$ with the Rost invariant of $E_{6}$ is the Rost invariant of $\mathrm{Sp}_{8}$.

Finally, we show that $H$ is adjoint, i.e., is $\mathrm{PGSp}_{8}$. Write $\rho: E_{6} \rightarrow G L_{27}$ for the 27-dimensional Weyl module of $E_{6}$ with highest weight $\omega_{1}$ (the fundamental dominant weight dual to $\alpha_{1}$ ); it is minuscule, so the representation is irreducible in all characteristics. By the explicit description of inclusion of coroot lattices from (5.2), we see that the restriction of $\omega_{1}$ to $\mathrm{Sp}_{8}$ is zero on $\check{\gamma}_{i}$ for $i \neq 2$ and is 1 on $\check{\gamma}_{2}$. That is, it restricts to the fundamental weight $\mu$ of the root system $C_{4}$ dual to $\gamma_{2}$. (This is standard, see e.g. [MP, p. 298].) Note that this weight belongs to the root lattice and so vanishes on the center of $\mathrm{Sp}_{8}$. Therefore, the center of $\mathrm{Sp}_{8}$ is in the kernel of the composition

$$
\mathrm{Sp}_{8} \rightarrow H \subset E_{6} \stackrel{\rho}{\rightarrow} G L_{27} .
$$

However, $\rho$ is injective, so it follows that the center of $\mathrm{Sp}_{8}$ is sent to zero in $H$. This proves that $H$ is adjoint.

5.4. Alternative definition of the invariant. We define an invariant $\Delta^{\prime}$ to be the composition

$$
\Delta^{\prime}: H^{1}\left(*, \mathrm{PGSp}_{8}\right) \rightarrow H^{1}\left(*, E_{6}\right) \rightarrow H^{3}(*, \mathbb{Q} / \mathbb{Z}(2)),
$$

where the first map comes from the inclusion $\mathrm{PGSp}_{8} \subset E_{6}$ and the second map is the Rost invariant of $E_{6}$. The image of the second map is contained in $H^{3}(*, \mathbb{Z} / 6 \mathbb{Z})$ by [GMS, p. 150]. 
Example 5.5. If $A$ is split, then $(A, \sigma)$ corresponds to the neutral class in $H^{1}\left(F, \mathrm{PGSp}_{8}\right)$. Since the maps in the definition of $\Delta^{\prime}$ are maps of pointed sets, $\Delta^{\prime}(A, \sigma)$ is zero.

Proposition 5.6. For every field $F$, the image of $\Delta^{\prime}$ lies in $H^{3}\left(F, \mu_{2}\right)$ and consists of symbols.

Proof. For every $(A, \sigma) \in H^{1}\left(F, \mathrm{PGSp}_{8}\right)$, there is an extension $K / F$ of degree a power of 2 that kills $(A, \sigma)$, hence also kills $\Delta^{\prime}(A, \sigma)$. It follows that the order of $\Delta^{\prime}(A, \sigma)$ belongs to the 2-primary torsion of $H^{3}(F, \mathbb{Z} / 6 \mathbb{Z})$, i.e., to $H^{3}\left(F, \mu_{2}\right)$.

Further, let $G$ be the simply connected group of type $E_{6}$ over $F$ obtained by twisting $E_{6}$ by the image of $(A, \sigma)$. As $G$ is split by $K$, it is split (hence $\Delta^{\prime}(A, \sigma)$ is zero) or has Tits index

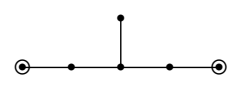

by [T, p. 58]. In the second case, the semisimple anisotropic kernel is a simply connected group of type ${ }^{1} D_{4}$ with trivial Tits algebras, i.e., $\operatorname{Spin}(q)$ for a 3-Pfister form $q$. It follows that $\Delta^{\prime}(A, \sigma)$ is the Arason invariant of $q$.

Example 5.7 (Hyperbolic involutions). Suppose that $\sigma$ is hyperbolic. We will prove that $\Delta^{\prime}(A, \sigma)$ is zero. Indeed, by [T, p. 56] or the kind of reasoning in [CG], the Tits index of $\operatorname{PGSp}(A, \sigma)$ is

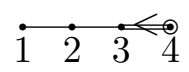

or has more vertices circled. That is, if we fix a set of roots of $\operatorname{PGSp}(A, \sigma)$ relative to a maximal $F$-torus containing a maximal $F$-split torus, the intersection of the kernels of the simple roots $\gamma_{1}, \gamma_{2}, \gamma_{3}$ contains a rank 1 split torus $S$, namely one corresponding to

$$
\check{\gamma}_{1}+2 \check{\gamma}_{2}+3 \check{\gamma}_{3}+4 \check{\gamma}_{4}
$$

in the coroot lattice.

Put $G$ for the group obtained by twisting $E_{6}$ by $(A, \sigma)$. The image of $S$ in $G$ corresponds to the coroot

$$
2 \check{\alpha}_{1}+2 \check{\alpha}_{2}+4 \check{\alpha}_{3}+6 \check{\alpha}_{4}+4 \check{\alpha}_{5}+2 \check{\alpha}_{6}
$$


by (5.2), i.e., to $2 \omega_{2}-2 \alpha_{2}$. The roots of $G$ that are orthogonal to $\omega_{2}-\alpha_{2}$ are those whose $\alpha_{2}$ and $\alpha_{4}$-coordinates agree, i.e., the linear combinations of the simple roots $\alpha_{1}, \alpha_{2}+\alpha_{4}, \alpha_{3}, \alpha_{5}, \alpha_{6}$. The centralizer $Z$ of $S$ in $G$ is reductive, and its semisimple part $Z^{\text {ss }}$ has root system of type $A_{5}$ consisting of the roots orthogonal to $\omega_{2}-\alpha_{2}$. Further, $Z^{\text {ss }}$ contains the semisimple anisotropic kernel of $G$.

Suppose for the sake of contradiction that $G$ is not split, hence the semisimple anisotropic kernel of $G$ is of type $D_{4}$. Over an algebraic closure of $F$, this gives an inclusion of split simply connected groups $D_{4} \subset A_{5}$, hence $D_{4}$ has a faithful representation of dimension 6 . But this is impossible. Therefore $G$ is split and the claim is proved.

We have:

The invariant $\Delta^{\prime}: H^{1}\left(*, \mathrm{PGSp}_{8}\right) \rightarrow H^{3}\left(*, \mu_{2}\right)$ is the invariant $\Delta$ given by Theorem $A$.

To see this, we note that $\Delta^{\prime}$ is an invariant, it satisfies property (1) of Theorem A by Example 5.7, and it is nonzero by (5.3). Therefore, $\Delta^{\prime}$ equals $\Delta$ by 4.5 .

\section{Square-Central Elements}

Let now $(A, \sigma)$ be a central simple $F$-algebra of degree 8 with symplectic involution. In this section, we find a $\sigma$-symmetric, trace zero element $u \in A$ such that $u^{2}$ belongs to $F^{\times}$.

Lemma 6.1. Suppose $A$ is division. Then:

(1) There exists an element $s \in A$ such that $\sigma(s)=-s$ and $s^{2} \in F^{\times}$.

(2) Any such element $s$ lies in a triquadratic extension $F\left(s_{1}, s_{2}, s_{3}\right) \subset A$ such that $\sigma\left(s_{i}\right)=-s_{i}$ and $s_{i}^{2} \in F^{\times}$for $i=1,2,3$ and $s=s_{1} s_{2} s_{3}$.

The lemma sharpens - and is proved using - a result of Rowen from [Row] (or see $[J, \S 5.6]$ ) that says that every $A$ as in the lemma contains a triquadratic extension of $F$. If $A$ is not division, there exist elements $s \in A$ such that $\sigma(s)=$ $-s, \operatorname{Trd}_{A}(s)=0$, and $s^{2} \in F^{\times}$; part (2) holds for any such element $s$. The proof is omitted, since this result is not used in the sequel.

We remark that part (1) of the lemma implies that $\sigma$ becomes hyperbolic over the quadratic extension $F(s)$ [BST, 3.3]. 
Proof. We first prove (1), which is the crux. Let $M$ be a triquadratic extension of $F$ contained in $A$; its existence is guaranteed by Rowen. Note that because $\sigma$ is symplectic and $M$ is a maximal subfield, $M$ cannot consist of $\sigma$-symmetric elements [KMRT, 4.13]. Therefore, there is some $a \in M$ such that $a^{2} \in F^{\times}$and $a$ is not fixed by $\sigma$. If $\sigma(a)=-a$, then we are done, so suppose not.

The elements $a$ and $\sigma(a)$ do not commute. Indeed, otherwise we would have:

$$
0=a^{2}-\sigma(a)^{2}=(a-\sigma(a))(a+\sigma(a))
$$

but neither of the terms in the product on the right are zero, contradicting the hypothesis that $A$ is division.

Let $Q$ be the $F$-subalgebra of $A$ generated by $a$ and $\sigma(a)$. (Note that $a$ is not in $F$ because $\sigma$ does not fix $a$.) The center of $Q$ contains the field $Z$ generated over $F$ by the element $z:=a \sigma(a)+\sigma(a) a$. As a $Z$-algebra, $Q$ is generated by $a$ and

$$
b:=\sigma(a)-a \sigma(a) a^{-1}=2 \sigma(a)-z a^{-1} .
$$

The element $b$ has square in $Z$ and anti-commutes with $a$, hence $Q$ is a quaternion algebra with center $Z$. By the original definition of $Q$, we see that $Q$ is stable under $\sigma$, hence $\sigma$ restricts to an involution of the first kind on $Q$. Further, $a$ has trace zero in $Q$, but $\sigma(a) \neq-a$, so $\sigma$ is orthogonal on $Q$.

We now consider the various cases for the dimension of $Z$ over $F$. We remark that $Z$ cannot be 4 -dimensional over $F$, because in that case the centralizer of $Z$ in $A$ is $Q$ itself, hence the restriction of $\sigma$ to $Q$ is symplectic, which is a contradiction.

Case $Z=F$ : In case $Z=F$, the algebra $A$ is the tensor product of $Q$ with the centralizer $C_{A}(Q)$ of $Q$ in $A$, and $C_{A}(Q)$ is a central simple $F$-algebra of degree 4. As $\sigma$ restricts to an orthogonal involution on $Q$, it restricts to a symplectic involution on $C_{A}(Q)$. But any biquaternion division algebra with symplectic involution contains a quaternion subalgebra such that the restriction of the symplectic involution is the canonical involution on the quaternion subalgebra [KMRT, 16.16]. In particular, $C_{A}(Q)$ has a square-central and skew-symmetric element, so we are done in this case.

Case $[Z: F]=2$ : By the Double Centralizer Theorem, the centralizer of $Z$ is a tensor product $Q \otimes_{Z} Q^{\prime}$ of $Q$ with another quaternion algebra $Q^{\prime}$. Further, the 
algebras $A \otimes_{F} Z$ and $C_{A}(Z)$ are Brauer-equivalent (over $Z$ ). Taking corestrictions of these two algebras, we find that

$$
\operatorname{cor}_{Z / F}[A \otimes Z]=2[A]=0
$$

which equals

$$
\operatorname{cor}_{Z / F}\left[C_{A}(Z)\right]=\operatorname{cor}_{Z / F}[Q]+\operatorname{cor}_{Z / F}\left[Q^{\prime}\right] .
$$

But we can write $Q$ as a quaternion algebra over $Z$ with one of the slots equal to $a^{2} \in F^{\times}$, so the corestriction of $Q$ has index at most 2 over $F$. Necessarily the same holds for the index of the corestriction of $Q^{\prime}$, so $Q^{\prime}$ contains a trace zero element $s$ whose square lies in $F$ [KMRT, 16.28]. But the restriction of $\sigma$ to $C_{A}(Z)$ is symplectic, so the restriction of $\sigma$ to $Q^{\prime}$ is also symplectic, hence $\sigma(s)=-s$, and we are done in this case.

The proof of (1) is now complete, and we use (1) to prove (2); let $s$ be the element provided by (1). The centralizer $C_{A}(s)$ of $s$ in $A$ is a central simple $F[s]$-algebra of degree 4 on which $\sigma$ restricts to be a unitary involution.

The discriminant algebra of $\left(C_{A}(s), \sigma\right)$ is $M_{3}(D)$ for some (possibly split) quaternion algebra $D$. The canonical involution on it is orthogonal, i.e., is adjoint to a skew-hermitian form $h$ on a rank $3 D$-module $V$. We decompose $V$ as an orthogonal sum of rank 1 subspaces $V_{1} \perp V_{2} \perp V_{3}$ and observe that the tensor product $\otimes_{i} C_{0}\left(V_{i},\left.h\right|_{V_{i}}\right)$ of even Clifford algebras is an $F$-subalgebra of $C_{0}(h)$, which is in turn isomorphic to $\left(C_{A}(s), \sigma\right)$ by the exceptional isomorphism $\mathrm{A}_{3}=\mathrm{D}_{3}$, cf. [KMRT, 15.24]. Note that $C_{0}\left(V_{i},\left.h\right|_{V_{i}}\right)$ is a quadratic extension $F\left[s_{i}\right]$ of $F$ where $s_{i}^{2}$ belongs to $F^{\times}$and the canonical involution on the even Clifford algebra maps $s_{i} \mapsto-s_{i}$, which gives (2) except for the claim that $s=s_{1} s_{2} s_{3}$. But the center of $C_{A}(s)$ is the quadratic extension $F[s]$, hence $s$ equals $\alpha s_{1} s_{2} s_{3}$ for some $\alpha \in F^{\times}$. Replacing $s_{1}$ by $\alpha s_{1}$ completes the proof of (2).

Corollary 6.2. Let $(A, \sigma)$ be a central simple F-algebra of degree 8 with symplectic involution. Then $A$ contains an element $u$ such that $\sigma(u)=u, u^{2} \in F^{\times}$, and $\operatorname{Trd}_{A}(u)=0$.

Proof. If $A$ is division, one takes $u$ to be the product $s_{1} s_{2}$, for elements $s_{1} s_{2}$ as in Lemma 6.1(2). The condition that $u$ has reduced trace zero follows from the fact that $A$ is division. 
If $A$ is not division, we write $A$ as $M_{2}(B)$, endow $B$ with a symplectic involution $\tau$, and view $\sigma$ as adjoint to a $\tau$-hermitian form on a rank $2 B$-vector space $V$. We decompose $V$ as an orthogonal sum of two rank 1 spaces $V_{1} \perp V_{2}$, and take $u \in A$ to be the element that fixes $V_{1}$ elementwise and acts as -1 on $V_{2}$.

\section{The 10-DIMENSIONAL QUADRATIC FORM $q_{u}$}

We continue the notation of $\S 6$ : $(A, \sigma)$ is a central simple $F$-algebra of degree 8 with symplectic involution.

7.1. Definition of $q_{u}$. Fix an element $u \in A$ as in Cor. 6.2. We use it to construct a quadratic form $q_{u}$.

The centralizer $C_{A}(u)$ is semisimple of dimension $2^{5}$ and center $F[u]$, and we may consider the reduced trace $\operatorname{Trd}_{C_{A}(u)}: C_{A}(u) \rightarrow F[u]$. Moreover, $\sigma$ restricts to a symplectic involution on $C_{A}(u)$. Consider the $F$-vector space

$$
V_{u}=\left\{x \in C_{A}(u) \mid \sigma(x)=x \text { and } \operatorname{Trd}_{C_{A}(u)}(x)=0\right\} .
$$

If $u^{2} \notin F^{\times 2}$ (i.e., $F[u]$ is a field), then $C_{A}(u)$ is a central simple $F[u]$-algebra of degree 4 , and it is proved in [KMRT, §15.C] that $x^{2} \in F[u]$ for $x \in V_{u}$. This property also holds when $u^{2} \in F^{\times 2}$ (see the proof of Prop. 7.2 below), so we may define a quadratic form

$$
q_{u}: V_{u} \rightarrow F
$$

as follows: let $t: F(u) \rightarrow F$ denote the $F$-linear map such that $t(1)=0$ and $t(u)=1$, and set

$$
q_{u}(x):=t\left(x^{2}\right) \quad \text { for } x \in V_{u} .
$$

Note that the dimension of $V_{u}$ - the vector space underlying $q_{u}$ - is 10 .

Proposition 7.2. $q_{u}$ is in $I^{3} F$.

As a consequence of the proposition, $q_{u}$ is isotropic (because its dimension is 10 [Lam, XII.2.8]), and so $q_{u}$ is Witt-equivalent to a multiple of a 3-Pfister form.

Proof of Prop. 7.2. (i): Suppose first that $u^{2}=\lambda^{2}$ for some $\lambda \in F^{\times}$, and let

$$
e_{1}=\frac{1}{2}\left(1-u \lambda^{-1}\right), \quad e_{2}=\frac{1}{2}\left(1+u \lambda^{-1}\right) .
$$


The elements $e_{1}, e_{2}$ are $\sigma$-symmetric idempotents such that $e_{1}+e_{2}=1$ and $\operatorname{Trd}_{A}\left(e_{1}\right)=\operatorname{Trd}_{A}\left(e_{2}\right)=4$. Therefore, letting $\tau_{1}$ (resp. $\left.\tau_{2}\right)$ denote the restriction of $\sigma$ to $e_{1} A e_{1}$ (resp. $e_{2} A e_{2}$ ), we have

$$
(A, \sigma)=\left(e_{1} A e_{1}, \tau_{1}\right) \boxplus\left(e_{2} A e_{2}, \tau_{2}\right),
$$

and $e_{1} A e_{1} \simeq e_{2} A e_{2}$ is of degree 4. Moreover,

$$
C_{A}(u)=C_{A}\left(e_{1}\right)=C_{A}\left(e_{2}\right)=\left(e_{1} A e_{1}\right) \times\left(e_{2} A e_{2}\right) .
$$

Letting $\operatorname{Sym}\left(\tau_{i}\right)^{0}=\left\{x \in \operatorname{Sym}\left(e_{i} A e_{i}, \tau_{i}\right) \mid \operatorname{Trd}_{e_{i} A e_{i}}(x)=0\right\} \quad(i=1,2)$, and denoting by $s_{i}: \operatorname{Sym}\left(\tau_{i}\right)^{0} \rightarrow F$ the quadratic form $s_{i}(x)=x^{2}$ (see [KMRT, $\S 15 . \mathrm{C}]$ ), we have

$$
V_{u}=\operatorname{Sym}\left(\tau_{1}\right)^{0} \times \operatorname{Sym}\left(\tau_{2}\right)^{0} \quad \text { and } \quad q_{u}=s_{1}-s_{2} .
$$

By [KMRT, 16.18], we have $q_{u} \in I^{3} F$.

(ii): By (i), we may assume $u^{2} \notin F^{\times 2}$. Then $C_{A}(u)$ is a central simple $F[u]$ algebra of degree 4 on which $\sigma$ restricts to a symplectic involution. By [KMRT, $\S 15 . \mathrm{C}], V_{u}$ is an $F[u]$-vector space of dimension 5 , endowed with the quadratic form

$$
s_{\sigma}(x)=x^{2} \in F[u] .
$$

By definition, $q_{u}$ is the Scharlau transfer:

$$
q_{u}=t_{*}\left(s_{\sigma}\right) .
$$

The quadratic form $\langle 1\rangle \perp-s_{\sigma}$ is an Albert form for $C_{A}(u)$ (see [KMRT, (16.8)]). In particular, it lies in $I^{2} F(u)$. By commutativity of the diagram

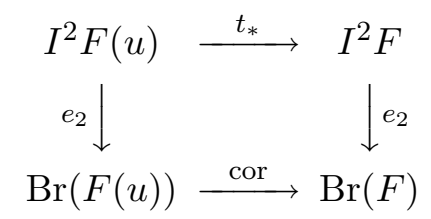

we get

$$
e_{2}\left(t_{*}\left(\langle 1\rangle \perp-s_{\sigma}\right)\right)=\operatorname{cor}_{F(u) / F}\left(C_{A}(u)\right) .
$$

Since $C_{A}(u)$ is Brauer-equivalent to $A \otimes_{F} F(u)$, and the Brauer class of $A$ is 2-torsion, it follows that

$$
e_{2}\left(t_{*}\left(\langle 1\rangle \perp-s_{\sigma}\right)\right)=0,
$$


hence $t_{*}\left(\langle 1\rangle \perp-s_{\sigma}\right) \in I^{3} F$ by a theorem of Pfister (note that this form has dimension 12), or of course by Merkurjev's theorem. Since $t_{*}(\langle 1\rangle)=0$, it follows that $q_{u}$ is in $I^{3} F$.

Example 7.3. Consider the special case where $(A, \sigma)$ is a tensor product

$$
\left(Q,{ }^{-}\right) \otimes\left(M_{2}(F), t\right) \otimes\left(M_{2}(F), \operatorname{Int}\left(\begin{array}{ll}
0 & 1 \\
1 & 0
\end{array}\right) t\right)
$$

where $Q$ is a quaternion algebra and $t$ denotes the transpose. For every $x \in F^{\times}$, the element $u:=1 \otimes 1 \otimes\left(\begin{array}{ll}0 & x \\ 1 & 0\end{array}\right)$ is $\sigma$-symmetric, has reduced trace zero, and has square $x$. We claim that $q_{u}$ is hyperbolic.

To see this, we put:

$$
v_{1}:=1 \otimes\left(\begin{array}{ll}
0 & 1 \\
1 & 0
\end{array}\right) \otimes 1 \quad \text { and } \quad v_{2}:=1 \otimes\left(\begin{array}{cc}
1 & 0 \\
0 & -1
\end{array}\right) \otimes 1 .
$$

The elements $v_{1}$ and $v_{2}$ belong to $V_{u}$ and are linearly independent over $F[u]$. For $c=c_{1} v_{1}+c_{2} v_{2}$ with $c_{1}, c_{2} \in F[u]$, we have $c^{2}=c_{1}^{2}+c_{2}^{2}$. The transfer of this 2-dimensional quadratic form $\langle 1,1\rangle$ is a direct sum of two hyperbolic planes, and we deduce that the anisotropic part of $q_{u}$ has dimension at most 6 . Since $q_{u}$ belongs to $I^{3} F$, we conclude that $q_{u}$ is hyperbolic.

\section{The Arason invariant of $q_{u}$ equals $\Delta(A, \sigma)$}

We continue the notation of $\S 6$ and $\S 7$ : $(A, \sigma)$ is a central simple $F$-algebra of degree 8 with symplectic involution, and $u$ is a $\sigma$-symmetric element of reduced trace zero such that $u^{2} \in F^{\times}$. The purpose of this section is to prove the following result, where $e_{3}$ denotes the Arason invariant $I^{3} F \rightarrow H^{3}\left(F, \mu_{2}\right)$.

Proposition 8.1. $e_{3}\left(q_{u}\right)=\Delta(A, \sigma)$.

Combining Propositions 8.1 and 7.2, we deduce that $\Delta(A, \sigma)$ is a symbol in $H^{3}\left(F, \mu_{2}\right)$, which recovers Prop. 5.6.

Proof of Prop. 8.1. (i): Suppose first that $F(u)$ is not a field. Using the notation of the proof of Prop. 7.2(i), we have

$$
(A, \sigma)=\left(e_{1} A e_{1}, \tau_{1}\right) \boxplus\left(e_{2} A e_{2}, \tau_{2}\right) \quad \text { and } \quad q_{u}=s_{1}-s_{2} .
$$

By [KMRT, 16.18], we have $e_{3}\left(s_{1}-s_{2}\right)=\Delta_{\tau_{1}}\left(\tau_{2}\right)$. On the other hand, Prop. 3.4 shows that $\Delta(A, \sigma)=\Delta_{\tau_{1}}\left(\tau_{2}\right)$. The proposition follows. 
For the rest of the proof, assume $F(u)$ is a field.

(ii): Suppose that $\tau$ is also a symplectic involution on $A$ that fixes $u$. We write $q_{u}^{\sigma}, V_{u}^{\sigma}$ and $q_{u}^{\tau}, V_{u}^{\tau}$ for the 10-dimensional quadratic forms and their underlying vector spaces over $F$ deduced from $A, \sigma, u$ and $A, \tau, u$ respectively as in 7.1 ; we will compute $e_{3}\left(q_{u}^{\sigma}-q_{u}^{\tau}\right)$.

Write $s_{u}^{\sigma}, s_{u}^{\tau}$ for the quadratic forms $x \mapsto x^{2}$ on $V_{u}^{\sigma}, V_{u}^{\tau}$ respectively, and also $\tilde{\sigma}, \tilde{\tau}$ for the restrictions of $\sigma, \tau$ to $\tilde{A}:=C_{A}(u)$. By the exceptional isomorphism $\mathrm{B}_{2}=\mathrm{C}_{2}$, the Arason invariant $e_{3}\left(s_{u}^{\sigma}-s_{u}^{\tau}\right)$ equals the relative invariant of symplectic involutions $\Delta_{\tilde{\tau}}(\tilde{\sigma})$, cf. [KMRT, 16.18]. Further, $\sigma=\operatorname{Int}(a) \tau$ for some $a \in A^{\times}$ that centralizes $u$, i.e., $a$ belongs to $\tilde{A}$. We have:

$$
e_{3}\left(s_{u}^{\sigma}-s_{u}^{\tau}\right)=\left(\operatorname{Nrp}_{\tilde{A}}(a)\right) \cdot[\tilde{A}] \quad \in H^{3}\left(F(u), \mu_{2}\right) .
$$

Taking the corestriction, we find:

$$
e_{3}\left(q_{u}^{\sigma}-q_{u}^{\tau}\right)=e_{3}\left(t_{*}\left(s_{u}^{\sigma}-s_{u}^{\tau}\right)\right)=\operatorname{cor}_{F(u) / F}\left(\left(\operatorname{Nrp}_{\tilde{A}}(a)\right) \cdot[\tilde{A}]\right) .
$$

But $\tilde{A}$ is Brauer-equivalent to $A \otimes_{F} F(u)$, so by the projection formula we have:

$$
e_{3}\left(q_{u}^{\sigma}-q_{u}^{\tau}\right)=\left(N_{F(u) / F} \operatorname{Nrp}_{\tilde{A}}(a)\right) \cdot[A]=\left(\operatorname{Nrp}_{A}(a)\right) \cdot[A]=\Delta_{\tau}(\sigma),
$$

where the middle equality is because $\operatorname{Nrp}$ is a square root of the reduced norm [KMRT, §2].

(iii): We now prove the proposition. If $A$ has index 1 or 2, then by Example 7.3 and Skolem-Noether, there is a hyperbolic symplectic involution $\tau$ on $A$ that fixes $u$, and the corresponding 10-dimensional quadratic form $q_{u}^{\tau}$ is hyperbolic. By (ii), we find:

$$
e_{3}\left(q_{u}^{\sigma}\right)=\Delta_{\tau}(\sigma)=\Delta(A, \sigma)
$$

If $A$ has index 4 or 8 , we can apply the Albert quadric method to conclude that $e_{3}\left(q_{u}^{\sigma}\right)$ equals $\Delta(A, \sigma)$.

\section{Proof of Theorem B: Detecting Decomposability}

We now prove Theorem $\mathrm{B}$, which asserts that $\Delta(A, \sigma)$ is zero if and only if $(A, \sigma)$ is completely decomposable. The "if" direction is Example 3.2. The "only if" direction holds if $A$ is not division by [BMT, Th. 8]. Thus, we only need to consider the case where $A$ is division. In that case, the theorem follows from Prop. 8.1 and the following more precise result: 
Proposition 9.1. Let $(A, \sigma)$ be a central division algebra of degree 8 with symplectic involution, and let $u \in A$ be such that $u^{2} \in F^{\times}, u \notin F$, and $\sigma(u)=u$.

(1) There is a biquadratic extension $K / F$ such that $u \in K \subset \operatorname{Sym}(A, \sigma)$.

(2) Let $K / F$ be an arbitrary extension of degree 4 such that $u \in K \subset$ $\operatorname{Sym}(A, \sigma)$. If $q_{u}$ is hyperbolic, there is a decomposition of $(A, \sigma)$ into a tensor product of quaternion algebras with canonical involutions

$$
(A, \sigma)=\left(Q_{1}, \gamma_{1}\right) \otimes_{F}\left(Q_{2}, \gamma_{2}\right) \otimes_{F}\left(Q_{3}, \gamma_{3}\right)
$$

such that $K \subset Q_{1} \otimes Q_{2}$.

Proof. For (1), we may take $K=F(u, v)$ where $v \in V_{u}$ is any isotropic vector of $q_{u}$.

For (2), suppose $K$ is an arbitrary quadratic extension of $F(u)$ in $\operatorname{Sym}(A, \sigma)$, and let $K=F(u)(x)$ where $x^{2} \in F(u)^{\times}$.

Suppose $q_{u}$ is hyperbolic, and let $U \subset V_{u}$ be a maximal totally isotropic $F$ subspace. For $y \in U \cap u U$, we have $u y \in U$, hence also $(1+u) y \in U$. Since $y$ and $(1+u) y$ are isotropic for $q_{u}$, we have

$$
y^{2} \in F \quad \text { and } \quad\left(1+2 u+u^{2}\right) y^{2} \in F .
$$

This is impossible if $y^{2} \neq 0$, hence $U \cap u U=\{0\}$ and therefore $V_{u}=U \oplus u U$.

Let $v, v^{\prime} \in U$ be such that

$$
x=v+u v^{\prime}
$$

Substituting $x u$ for $x$ if necessary, we may assume $v \neq 0$. If $v$ and $v^{\prime}$ are linearly dependent over $F$, pick any nonzero $w \in v^{\perp} \cap U$. Otherwise, let

$$
w:=v^{\prime}-v v^{\prime} v^{-1}=2 v^{\prime}-v \frac{v v^{\prime}+v^{\prime} v}{v^{2}} .
$$

(Note that $w$ is nonzero by the second expression for $w$, because $v$ and $v^{\prime}$ are linearly independent.) Since $v, v^{\prime}$, and $v+v^{\prime}$ are isotropic for $q_{u}$, we have $v^{2}, v^{\prime 2}$, $v v^{\prime}+v^{\prime} v \in F$, hence $w \in U$ and $w^{2} \in F^{\times}$. Moreover, $w$ anticommutes with $v$, and the $F(u)$-span of $v$ and $w$ contains $x$. Thus, in both cases $v$ and $w$ generate a quaternion $F$-algebra $H$ stable under $\sigma$, and $K \subset H \otimes_{F} F(u)$. The restriction of $\sigma$ to $H$ is an orthogonal involution since $\sigma(v)=v$ and $\sigma(w)=w$. Therefore, the restriction of $\sigma$ to the centralizer $C_{A}(H)$ is symplectic. By [KMRT, (16.16)], 
there is a decomposition into quaternion $F$-algebras

$$
\left(C_{A}(H), \sigma\right)=\left(H^{\prime}, \sigma^{\prime}\right) \otimes_{F}\left(Q_{3}, \gamma_{3}\right)
$$

where $u \in H^{\prime}, \sigma^{\prime}$ is an orthogonal involution and $\gamma_{3}$ is the canonical involution, hence

$$
(A, \sigma)=\left(H,\left.\sigma\right|_{H}\right) \otimes\left(H^{\prime}, \sigma^{\prime}\right) \otimes\left(Q_{3}, \gamma_{3}\right) .
$$

Now, we can find quaternion algebras $Q_{1}, Q_{2}$ with canonical involutions $\gamma_{1}, \gamma_{2}$ such that

$$
\left(H,\left.\sigma\right|_{H}\right) \otimes\left(H^{\prime}, \sigma^{\prime}\right)=\left(Q_{1}, \gamma_{1}\right) \otimes\left(Q_{2}, \gamma_{2}\right) .
$$

Since $K=F(u, x) \subset H \otimes H^{\prime}=Q_{1} \otimes Q_{2}$, the proof is complete.

\section{Application to the Pfister Factor Conjecture}

We now use our invariant to give an alternate proof of the Pfister Factor Conjecture for tensor products of 6 quaternion algebras.

Let $Q_{1}, \ldots, Q_{6}$ be quaternion $F$-algebras with canonical involutions $\gamma_{1}, \ldots$, $\gamma_{6}$. Assume $\bigotimes_{i=1}^{6} Q_{i}$ is split and let $\varphi$ be a $2^{6}$ dimensional quadratic form representing 1 such that

$$
\gamma_{1} \otimes \cdots \otimes \gamma_{6}=\operatorname{ad}_{\varphi}
$$

We have to show that $\varphi$ is a Pfister form.

Put $A:=Q_{1} \otimes Q_{2} \otimes Q_{3}$ and $s=\gamma_{1} \otimes \gamma_{2} \otimes \gamma_{3}$. The case where $A$ is not division is handled by [ST, Lemma 2], so we assume below that $A$ is division.

Since $\otimes_{i=1}^{6} Q_{i}$ is split, we may identify $A$ also with $Q_{4} \otimes Q_{5} \otimes Q_{6}$. Let $\sigma:=$ $\gamma_{1} \otimes \gamma_{2} \otimes \gamma_{3}$ and $\tau:=\gamma_{4} \otimes \gamma_{5} \otimes \gamma_{6}$, and let $s \in \operatorname{Sym}(A, \sigma)$ be such that

$$
\tau=\operatorname{Int}(s) \sigma
$$

so that

$$
\operatorname{ad}_{\varphi}=\sigma \otimes \tau=\sigma \otimes(\operatorname{Int}(s) \sigma) .
$$

Note that we may assume that $F(s)$ is a quadratic or biquadratic extension of $F$. Indeed, we may find an odd-degree extension $E / F$ such that $F(s) \otimes_{F} E$ is a 2-extension of $E$. (Take for $E$ the cubic resolvent of $F(s)$ if $s$ has degree 4.) If we show that $\varphi_{E}$ is a Pfister form, then an easy argument (see [Rost]) shows that $\varphi$ also is a Pfister form. Therefore, substituting $E$ for $F$, we may henceforth assume $F(s) / F$ is quadratic or biquadratic. 
Further, we may assume that $s$ belongs to $Q_{1} \otimes Q_{2} \otimes 1$. Let $u \in F(s) \backslash F$ be a square-central element. Since $\sigma$ is decomposable, Example 3.2 and Prop. 8.1 show that $q_{u}$ is hyperbolic, hence, by Proposition 9.1, we may find a decomposition

$$
(A, \sigma)=\left(Q_{1}^{\prime}, \gamma_{1}^{\prime}\right) \otimes\left(Q_{2}^{\prime}, \gamma_{2}^{\prime}\right) \otimes\left(Q_{3}^{\prime}, \gamma_{3}^{\prime}\right)
$$

such that $s \in Q_{1}^{\prime} \otimes Q_{2}^{\prime}$. Replacing the $Q_{i}$ with $Q_{i}^{\prime}$ for $i=1,2,3$, we may assume that $s$ belongs to $Q_{1} \otimes Q_{2}$ so that

$$
\tau=\left(\operatorname{Int}(s)\left(\gamma_{1} \otimes \gamma_{2}\right)\right) \otimes \gamma_{3} .
$$

Writing $n_{i}$ for the norm form of $Q_{i}$, we have:

$\otimes_{i}\left(Q_{i}, \gamma_{i}\right)=\left(Q_{1}, \gamma_{1}\right) \otimes\left[\left(Q_{2}, \gamma_{2}\right) \otimes\left(Q_{1} \otimes Q_{2}, \operatorname{Int}(s)\left(\gamma_{1} \otimes \gamma_{2}\right)\right)\right] \otimes\left(Q_{3}, \gamma_{3}\right) \otimes\left(Q_{3}, \gamma_{3}\right)$.

The involution in brackets is a symplectic involution on $Q_{2} \otimes Q_{1} \otimes Q_{2}$, so it is adjoint to a hermitian form over $\left(Q_{1}, \gamma_{1}\right)$. Using a diagonalization of this form, we get a 4 -dimensional quadratic form $\psi$ over $F$ representing 1 such that

$$
\left(Q_{2}, \gamma_{2}\right) \otimes\left(Q_{1} \otimes Q_{2}, \operatorname{Int}(s)\left(\gamma_{1} \otimes \gamma_{2}\right)\right) \cong\left(M_{4}(F), \operatorname{ad}_{\psi}\right) \otimes\left(Q_{1}, \gamma_{1}\right) .
$$

Comparing the value of $\Delta$ on each side, we obtain

$$
\left[Q_{2}\right] \cdot\left(\operatorname{Nrd}_{Q_{1} \otimes Q_{2}}(s)\right)=\left[Q_{1}\right] \cdot(\operatorname{disc} \psi) .
$$

Since

$$
\left(\left[Q_{1}\right]+\left[Q_{2}\right]\right) \cdot\left(\operatorname{Nrd}_{Q_{1} \otimes Q_{2}}(s)\right)=\left[Q_{1} \otimes Q_{2}\right] \cdot\left(\operatorname{Nrd}_{Q_{1} \otimes Q_{2}}(s)\right)=0,
$$

it follows that

$$
\left[Q_{1}\right] \cdot(\operatorname{disc} \psi)=\left[Q_{1}\right] \cdot\left(\operatorname{Nrd}_{Q_{1} \otimes Q_{2}}(s)\right)
$$

Plugging (10.3) into (10.2), we find that $\varphi$ is isomorphic to $n_{1} \otimes n_{3} \otimes \psi$, and in particular belongs to $I^{5} F$. We evaluate the invariant $e_{5}: I^{5} F / I^{6} F \rightarrow H^{5}\left(F, \mu_{2}\right)$ on $\varphi$ and find:

$$
e_{5}(\varphi)=\left[Q_{1}\right] \cdot\left[Q_{3}\right] \cdot(\operatorname{disc} \psi)
$$

But $\tau$ is decomposable, so (10.1) yields:

$$
\left[Q_{3}\right] \cdot\left(\operatorname{Nrd}_{Q_{1} \otimes Q_{2}}(s)\right)=\Delta(A, \tau)=0 .
$$

Combining equations (10.4) through (10.6) gives that $e_{5}(\varphi)$ is zero. That is, $\varphi$ belongs to $I^{6} F$. Therefore, $\varphi$ must be a 6 -fold Pfister form since $\operatorname{dim} \varphi=2^{6}$. This completes the proof. 


\section{A QUESTION}

Given a central simple algebra $A$ of exponent 2 and degree $2 n$, with $n$ divisible by 4 , one can define a corresponding element of the torsion of the Chow group $\mathrm{CH}^{2}\left(X_{A}\right)$ as follows, where $X_{A}$ denotes the Severi-Brauer variety of $A$.

Choose a symplectic involution $\sigma$ on $A$. The discriminant $\Delta(A, \sigma)$ is in the kernel of the map

$$
\text { res: } H^{3}\left(F, \mu_{2}\right) \rightarrow H^{3}\left(F\left(X_{A}\right), \mu_{2}\right) .
$$

Moreover, the class of $\Delta(A, \sigma)$ in

$$
\operatorname{ker}(\mathrm{res}) /[A] \cdot H^{1}\left(F, \mu_{2}\right)
$$

depends only on $A$ and not on the choice of $\sigma$; we denote this class by $\Delta(A)$.

The quotient group (11.1) is identified with the torsion $\mathrm{CH}^{2}\left(X_{A}\right)_{\text {tor }}$ in the Chow group $\mathrm{CH}^{2}\left(X_{A}\right)$, see $[\mathrm{P}]$, and we may view $\Delta(A)$ as belonging to this group. By a theorem of Karpenko [Kar, 5.3], this group is trivial if $A$ is decomposable of degree 8 . However, for the generic algebra $A^{\text {gen }}$ of degree 8 and exponent 2 , the group $\mathrm{CH}^{2}\left(X_{A^{\text {gen }}}\right)_{\text {tor }}$ is $\mathbb{Z} / 2 \mathbb{Z}$ by $[$ Kar, 5.1].

Question 11.2. Is $\Delta\left(A^{\text {gen }}\right)$ nonzero in $\mathrm{CH}^{2}\left(X_{A \text { gen }}\right)_{\text {tor }}$ ? More generally, is $\Delta(A)$ nonzero for $A$ indecomposable of degree 8 ?

\section{REFERENCES}

[A] J.Kr. Arason, Cohomologische invarianten quadratischer Formen, J. Algebra 36 (1975), 448-491.

[Be] K. Becher, A proof of the Pfister Factor Conjecture, Invent. Math. (2008), DOI $10.1007 / \mathrm{s} 00222-007-0107-5$

[BMT] G. Berhuy, M. Monsurrò, and J.-P. Tignol, The discriminant of a symplectic involution, Pacific J. Math. 209 (2003), 201-218.

[BST] E. Bayer-Fluckiger, D.B. Shapiro, and J.-P. Tignol, Hyperbolic involutions, Math. Z. 214 (1993), no. 3, 461-476.

[CG] M. Carr and S. Garibaldi, Geometries, the principle of duality, and algebraic groups, Expo. Math. 24 (2006), 195-234.

[D] E.B. Dynkin, Semisimple subalgebras of semisimple Lie algebras, Amer. Math. Soc. Transl. (2) 6 (1957), 111-244, [Russian original: Mat. Sbornik N.S. 30(72) (1952), 349-462].

[GMS] S. Garibaldi, A.S. Merkurjev, and J-P. Serre, Cohomological invariants in Galois cohomology, University Lecture Series, vol. 28, Amer. Math. Soc., 2003.

[J] N. Jacobson, Finite-dimensional division algebras over fields, Springer, 1996. 
[Kahn] B. Kahn, Lower $\mathcal{H}$-cohomology of higher-dimensional quadrics, Arch. Math. (Basel) 65 (1995), no. 3, 244-250.

[Kar] N.A. Karpenko, Codimension 2 cycles on Severi-Brauer varieties, K-Theory 13 (1998), no. 4, 305-330.

[KMRT] M.-A. Knus, A.S. Merkurjev, M. Rost, and J.-P. Tignol, The book of involutions, Colloquium Publications, vol. 44, Amer. Math. Soc., 1998.

[KPS] M.-A. Knus, R. Parimala, and R. Sridharan, Pfaffians, central simple algebras and similitudes, Math. Z. 206 (1991), 589-604.

[KQ] N. Karpenko and A. Quéguiner, A criterion of decomposability for degree 4 algebras with unitary involution, J. Pure Appl. Algebra 147 (2000), 303-309.

[Lam] T.Y. Lam, Introduction to quadratic forms over fields, Graduate Studies in Mathematics, vol. 67, Amer. Math. Soc., Providence, RI, 2005.

[Mac] M. MacDonald, Cohomological invariants of odd degree Jordan algebras, preprint, 2007.

[MP] W.G. McKay and J. Patera, Tables of dimensions, indices, and branching rules for representations of simple Lie algebras, Lecture Notes in Pure and Applied Mathematics, vol. 69, Marcel Dekker Inc., New York, 1981.

[P] E. Peyre, Galois cohomology in degree three and homogeneous varieties, $K$-Theory 15 (1998), no. 2, 99-145.

[Rost] M. Rost, A descent property for Pfister forms, J. Ramanujan Math. Soc. 14 (1999), no. $1,55-63$.

[Row] L.H. Rowen, Central simple algebras, Israel J. Math. 29 (1978), no. 2-3, 285-301.

[Sh] D.B. Shapiro, Compositions of quadratic forms, Expositions in Mathematics, vol. 33, de Gruyter, Berlin, 2000.

[ST] A. Serhir and J.-P. Tignol, The discriminant of a decomposable symplectic involution, J. Algebra 273 (2004), 601-607.

[St end] R. Steinberg, Endomorphisms of linear algebraic groups, Mem. Amer. Math. Soc. 80 (1968), 1-108, (= Collected Papers \#23).

[St lect] _ Lectures on Chevalley groups, Yale University, New Haven, Conn., 1968.

[St coll $]$ _ Collected papers, Amer. Math. Soc., Providence, RI, 1997.

[T] J. Tits, Classification of algebraic semisimple groups, Algebraic Groups and Discontinuous Subgroups, Proc. Symp. Pure Math., vol. IX, AMS, 1966, pp. 32-62.

Skip Garibaldi

Department of Mathematics \& Computer Science

Emory University

Atlanta, Georgia 30322

USA

E-mail: skip@member.ams.org

http://www.mathcs.emory.edu/ ${ }^{\sim}$ skip/ 
R. Parimala

Department of Mathematics \& Computer Science

Emory University

Atlanta, Georgia 30322

USA

E-mail: parimala@mathcs.emory.edu

http://www.mathcs.emory.edu/ parimala/

Jean-Pierre Tignol

Département de mathématique

Université catholique de Louvain

chemin du cyclotron, 2

B-1348 Louvain-la-Neuve

Belgium

E-mail: jean-pierre.tignol@uclouvain.be

http://www.math.ucl.ac.be/membres/tignol/ 